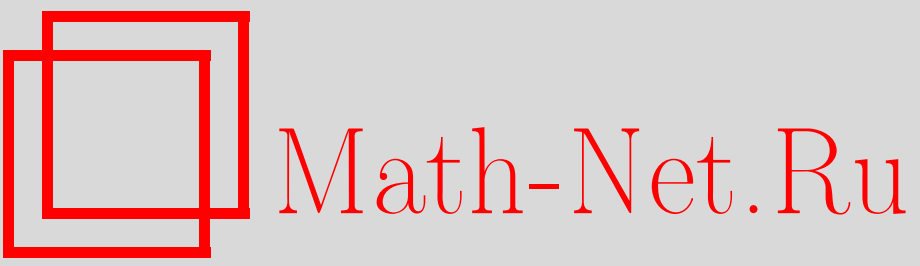

В. М. Максимов, Представление алгебры линейных отображений косым произведением алгебр, УМН, 2001, том 56, выпуск 1, 171-172

DOI: https://doi.org/10.4213/rm369

Использование Общероссийского математического портала Math-Net.Ru подразумевает, что вы прочитали и согласны с пользовательским соглашением

http://www.mathnet.ru/rus/agreement

Параметры загрузки:

IP: 52.6 .47 .48

26 апреля 2023 г., 13:46:40 


\title{
ПРЕДСТАВЛЕНИЕ АЛГЕБРЫ ЛИНЕЙНЫХ ОТОБРАЖЕНИЙ КОСЫМ ПРОИЗВЕДЕНИЕМ АЛГЕБР
}

\author{
В. М. МАКСИМОВ
}

1. Конструкции косых полиномов, рядов [1]-[4], косых алгебр таких, как алгебры Клиффорда и их обобщения [5], [6], а также аналогичных конструкций для алгебр Хопфа [6] являются предметом постоянных исследований, так как находят широкое применение [7], [8].

В частности, представления алгебры линейных отображений пространства полиномов в себя косыми рядами по степеням $D$ - обычных операторов дифференцирования [9], лежит в основе классического теневого исчисления (см. [10], [11]). Обобщения этого исчисления см. в [12]-[15].

В данном сообщении мы даем общую теорему о представлении алгебры $\mathscr{A}(L)$ всех линейных отображений линейного пространства $L$ над полем $\mathscr{F}($ для удобства char $\mathscr{F}=0)$ косьм произведением алгебр. Мы полагаем, что $\operatorname{dim} L=|L|$ конечна или счетна.

Следует отметить, что разложение Тейлора (1715 г.) $E^{y}=\sum y^{n} D^{n} / n$ !, где $E^{y}$ - оператор сдвига переменной на $y$, и разложение Бернулли (1694 г.) $V_{0}=\sum(-1)^{n} X^{n} D^{n} / n$ !, где $V_{0}-$ оператор значения в нуле, $X$ - оператор умножения на переменную, дают примеры разложения этих операторов при таком их представлении.

ОпредЕлЕниЕ 1 . Ассоциативную алгебру $S$ над полем $\mathscr{F}$ будем называть косым произведением алгебр $A$ и $B$ над $\mathscr{F}$, если $S$ содержит подалгебры $\mathscr{A}$ и $\mathscr{B}$, изоморфные соответственно $A$ и $B$, и если каждый элемент $s \in S$ может быть однозначно представлен в виде конечной или бесконечной суммы $s=\alpha_{0}+\alpha_{1} b_{1}+\alpha_{2} b_{2}+\cdots$, где $\alpha_{i} \in \mathscr{A}$ определяются $s$, a $b_{1}, b_{2}, \ldots-$ некоторый базис $\mathscr{B}$.

Базис $\left\{b_{k}\right\}$ определяет операторы $\psi_{j}^{(i)}$ алгебры $\mathscr{A}$ по правилу $b_{i} \alpha=\psi_{0}^{(i)}(\alpha)+\psi_{1}^{(i)}(\alpha) b_{1}+\cdots$ для каждого $\alpha \in \mathscr{A}$.

Если структурные константы, определяемые базисом $\left\{b_{k}\right\}$, и операторы $\psi_{j}^{(i)}$ удовлетворяют соотношениям, вытекающим из условий ассоциативности, то алгебра $\mathscr{B}$ и операторы $\psi_{j}^{(i)}$ определяют алгебру $S$ однозначно.

Заметим, что косое произведение алгебр можно также описать как скрещенное произведение этих алгебр (см., например, [6]).

2. ОПРеДЕлЕниЕ 2. Всякую алгебру $\mathscr{T}$ операторов из $\mathscr{A}(L)$ будем называть алгеброй восстановления базиса $\left\{l_{k}\right\}, k=0,1,2, \ldots$, пространства $L$, если в $\mathscr{T}$ имеется линейньй базис $\left\{\Gamma_{k}\right\}$, $k=0,1,2, \ldots$, такой, что $\Gamma_{k} l_{0}=l_{k}$.

ОПРЕДЕЛЕниЕ 3 . Всякую алгебру $\mathscr{B}$ операторов из $\mathscr{A}(L)$ будем назьвать локальной относителшно базиса $\left\{l_{k}\right\}, k=0,1,2, \ldots$, пространства $L$, если в $\mathscr{B}$ имеется базис $\left\{B_{k}\right\}$ такой, что вьполнены следующие равенства:

$$
B_{k} l_{i}=0, \quad i<k, \quad B_{k} l_{k}=l_{0} \quad \text { (условия локальности). }
$$

Теорема 1 (о разложении операторов). Каждый оператор $T \in \mathscr{A}(L)$ однозначно представляется в виде

$$
T=\gamma_{0}+\gamma_{1} B_{1}+\gamma_{2} B_{2}+\cdots,
$$

где $\gamma_{i} \in \mathscr{T}-$ из определения $2, a\left\{B_{k}\right\}-$ базис из определения 3.

В силу (1) лишь конечное число значений операторов $B_{k}$ ряда (2) отлично от нуля на любом базисном элементе $l_{k}$. Поэтому сумма $(2)$ может быть конечной и бесконечной. 
3. ОпределЕниЕ 4. Алгебру $\mathscr{P}$ над полем $\mathscr{F}$ будем называть обобщенно локальной, если в ней имеется базис $\left\{P_{k}\right\}$, для которого выполнены условия:

1) если произведение $P_{i} \cdot P_{j} \neq 0$, то при разложении по базису $\left\{P_{k}\right\}$ оно содержит только элементы $\operatorname{Pr}$ с $r>j$;

2) для любых целых $d>0$ и $j>0$ найдется целое $\delta(d, j)>0$ такое, что для $n>\delta(d, j)$ в разложении $P_{n} P_{j}$ по базису $\left\{P_{k}\right\}$ встречаются только элементы $P_{r} \mathrm{c} r>d$, либо $P_{n} P_{j}=0$.

Очевидно, градуированные алгебры $\left(\operatorname{deg} P_{i}=i\right)$ являются обобщенно локальными.

ТеОРема 2. Если дана произвольная обобщенно локальная алгебра ЯР, определяемая в базисе $\left\{P_{k}\right\}$ структурными константами $\left\{C_{k}^{i j}\right\}$, то для любого базиса $\left\{l_{k}\right\}$ пространства $L$ операторы $\left\{B_{k}\right\}$, определяемье условиями:

1) $B_{k} l_{i}=0, i<k, B_{k} l_{k}=l_{0}$ (свойство локальности),

2) $B_{n} l_{n+m}=\beta_{1}^{n, m} l_{1}+\beta_{2}^{n, m} l_{2}+\cdots$, где $\beta_{k}^{n, m}=C_{n+m}^{k, m}$,

порождают локальную алгебру $\mathscr{B}$, изоморфную алгебре $\mathscr{P}$ (причем в силу локальности сумма в 2) конечна).

Доказательство основано на формальной проверке изоморфизма при соответствии $P_{i} \rightarrow B_{i}$.

ПредЛожениЕ 1. Для каждой алгебры $\mathscr{G}$ над полем $\mathscr{F},|\mathscr{G}|=|L|$, и любого базиса $\left\{l_{k}\right\}$ пространства $L$ существует изоморфная ей алгебра операторов восстановления $\mathscr{T}$.

Таким образом, из теорем 1,2 и предложения 1 вытекает

ТЕОРема 3 (о косом представлении). Пусть $\mathscr{G}$ - произвольная алгебра над $\mathscr{F},|\mathscr{G}|=|L|$, и $\mathscr{B}$ - произвольная обобщенно локальная алгебра над $\mathscr{F},|\mathscr{B}|=|L|-1$. Тогда алгебра $\mathscr{A}(L)$ изоморфна некоторому косому произведению алгебр $\mathscr{G}$ и $\mathscr{B}$.

Частные случаи этой теоремы при $|L|=2^{q},|L|<\infty$ и счетном $L$ были рассмотрены в [9], $[14],[16],[17]$.

\section{СПИСОК ЛИТЕРАТУРЫ}

[1] O. Ore // Ann. of Math. (2). 1933. V. 34. P. 480-508. [2] F. Dumas // Lecture Notes in Math. 1991. V. 1478. Р. 192-214. [3] В. М. Максимов // УМН. 2000. Т. 55. № 4. С. 215-216. [4] T. H. M. Smits // Proc. Nederl. Akad. Wet. Ser. A. 1968. V.71. P. 209-224. [5] A. K. Kwasnewski // J. Phys. A. 1986. V. 19. P. 1469-1476. [6] К. Кассельс. Квантовые группы. Москва: Фазис, 1999. [7] Б.И. Ботвинник, В.М. Бухштабер, С.П. Новиков, С. А. Юзвинский // УМН. 2000. Т. 55. № 4. С. 5-24. [8] A. Bottreau, A. Di Bucchianico, D. E. Loeb // Discrete Math. 1998. V. 180. P. 65-75. [9] C. Г. Курбанов, В. M. Максимов // Докл. УзССР. 1986. № 4. C. 809-811. [10] G.-C. Rota. Finite Operator Calculus. London: Academic Press, 1975. [11] S. M. Roman. The Umbral Calculus. London: Academic Press, 1984. [12] О. В. Висков // Докл. АН СССР. 1978. Т. 239. С. 22-25. [13] О. В. Висков // Труды МИАН. 1986. Т. 177. С. 21-32. [14] О.В. Висков. Обобщенные функции и их применения в математической физике // Труды Межд. конф. АН СССР. Москва: ВЦ, 1981. С. 111-120. [15] A. K. Kwasnewski // Preprint № 5. Poland: Univ. Bialystok, 2000. [16] В. M. Максимов // Логико-алгебраические конструкции: Изд-во Калининского гос. ун-та, 1987. С. 61-65. [17] В.М. Максимов // Алгебраические и логические конструкции: Изд-во Калининского гос. ун-та, 1989. С. $72-77$. 